\title{
Multipole decomposition of the cross section of the neutrino-lead scattering process ${ }^{208} \mathrm{~Pb}($ g.s. $)\left(v, v^{\prime}\right){ }^{208} \mathrm{~Pb}^{*}$

\author{
O. Civitarese $\mathrm{e}^{1,2, *}$ and T. Tarutina ${ }^{2}$ \\ ${ }^{1}$ Departament of Physics, University of La Plata, C.C. 67 1900, La Plata, Argentina \\ ${ }^{2}$ IFLP (CONICET) La Plata, Argentina
}

(Received 13 July 2016; revised manuscript received 20 September 2016; published 2 November 2016)

\begin{abstract}
We calculate neutral-current contributions to the process $v+{ }^{208} \mathrm{~Pb}$ (g.s) $\longrightarrow v^{\prime}+{ }^{208} \mathrm{~Pb}^{*}$, for incoming neutrino energies up to $150 \mathrm{MeV}$. The spectrum of ${ }^{208} \mathrm{~Pb}$ is calculated by performing a diagonalization of the $\delta$-force interaction in the space of particle-hole pairs, both for neutrons and protons. We study the dependence of the cross section on the energy of the neutrino and present its multipole decomposition for normal and abnormal parity states. We compare our results to the estimate of neutral-current contributions by other authors, and to our previous results for the charged-current channel.
\end{abstract}

DOI: 10.1103/PhysRevC.94.054603

\section{INTRODUCTION}

Neutrino-related reactions and decays are relevant for a significant number of physical processes on a broad energy domain. They are found in the chain of reactions leading to primordial elements [1], in supernova explosions [2], as well as in exotic electro-weak decays [3]. The experimental observations of these phenomena have greatly advanced the present knowledge about neutrino flavor oscillations and neutrino interactions and established limits on the neutrino mass and possible mass hierarchies [4]. The measurement of neutrino-nucleus interactions has been advocated in a number of papers, particularly in Refs. [5-8] where large-scale calculations of the corresponding cross sections have been reported. Elliott [5] has presented predictions for measurements of supernova neutrinos using lead perchlorate and shows that such a detector can be sensitive to neutrino-induced reactions of various types, i.e., reactions mediated by neutral and charged currents, secondary neutron and gamma-rays emission. Along this line, the works of Fuller, Haxton, and McLaughlin [6], and Engel, McLaughlin, and Volpe [7] advanced the notion about the convenience of a lead-based detector as a proper instrument for the study of supernova neutrinos. Similar arguments were presented in a paper by Kolbe and Langanke [8]. The interest on lead-based detectors has been renewed by the HALO (Helium and Lead Observatory) project at SNOLAB [9].

We have taken these previous papers and the experimental interest on the neutrino-lead interactions as a motivation to perform a detailed calculation of the nuclear response of $\mathrm{Pb}$ to neutrino probes. In Ref. [10] the charged-current contribution to the neutrino-nucleus interaction, for the same nuclear target ${ }^{208} \mathrm{~Pb}$, was studied. The estimation of the cross section, for that case, requires the knowledge of matrix elements of isovector transition operators connecting the ground state of ${ }^{208} \mathrm{~Pb}$ with the excited states of ${ }^{208} \mathrm{Bi}$. In Ref. [10] proton single-particle states were taken to be bound, quasibound, resonant, and continuum states, and the wave functions for

*osvaldo.civitarese@ fisica.unlp.edu.ar states of ${ }^{208} \mathrm{Bi}$ were calculated in that extended basis, both to evaluate the effects of resonant and continuum states on the cross section, as well as to study the dependence of the results on the chosen residual proton-neutron interaction. The calculated cross section for $q=100 \mathrm{MeV}$, where $q$ is the momentum transferred by the neutrino, was of the order of $\sigma \approx 3.04 \times 10^{-38} \mathrm{~cm}^{2}$.

The present work is a continuation of the work done in Ref. [10], and it is aimed at the calculation of the response of a lead-based detector to the neutral-currents sector of the neutrino-nucleus interaction. Such a calculation will then allow us to evaluate the ratio of $\sigma\left(\nu, v^{\prime}\right)$ with respect to the charged-currents one $\sigma\left(v, e^{-}\right)$, and then get an estimate of the total neutrino-nucleus cross section. We started from the nuclear structure sector of the calculation, and wrote the wave function of states in ${ }^{208} \mathrm{~Pb}$ as combinations of neutron and proton particle-hole configurations. We have chosen the $\delta$-force residual interaction to calculate the matrix elements of the nuclear Hamiltonian, and extracted the corresponding eigenvalues and eigenvectors from a direct diagonalization of the interaction. As we shall show, while presenting our results, the choice of the interaction does not affect substantially the value of the cross section at a given incoming neutrino energy. This is supported by a comparison between the present results and other results available in the literature.

With these wave functions we have calculated the scattering amplitudes and the corresponding cross section as a function of the energy of the incoming neutrino. Then we focus on the contribution of different multipoles to the cross section, and compare our predictions on neutral-current contributions with the predictions of earlier works [5-8]. This was done to assess the independence of the results upon the choice of a particular two-body interaction.

The paper is organized as follows. The formalism is described in Sec. II, where the details of the neutral-current neutrino-nucleus interactions are given in Sec. II A, and the nuclear structure details in Sec. IIB. The results of the calculations are presented and discussed in Sec. III. Finally, our conclusions are drawn in Sec. IV. 


\section{FORMALISM}

A comprehensive description of neutrino-nucleus interaction at low energies was presented in the book of Blin-Stoyle [11], and later applied to the calculation of various processes involving neutrinos. The formalism for the case of charged-current neutrino nucleus interactions was presented in Refs. [12-14]. Here, because the theory is rather well known, we shall briefly review the essentials needed for the calculations.

\section{A. Neutral-current neutrino-nucleus interactions}

The cross section for the inelastic scattering of neutrinos by lead: $v+{ }^{208} \mathrm{~Pb} \longrightarrow v^{\prime}+{ }^{208} \mathrm{~Pb}^{*}$ is given by the formula

$$
\begin{aligned}
\sigma= & (2 \pi)^{4} \sum_{f} \int d^{3} p_{v_{f}} \delta\left(E_{v_{f}}+E_{f}-E_{v_{i}}-E_{i}\right) \\
& \times\left|\left\langle v_{f}\left(p_{v_{f}}\right) ; f\left|H_{\mathrm{eff}}\right| v_{i}\left(p_{v_{i}}\right) ; i\right\rangle\right|^{2},
\end{aligned}
$$

where $E_{i}$ is the energy of the target nucleus, $E_{f}$ is the final energy of the residual nucleus, that is the energy of one of the excited states of ${ }^{208} \mathrm{~Pb}, E_{v_{i}}$ is the energy of the incoming neutrino, and $E_{v_{f}}$ is the energy of the outgoing neutrino. The ket $\left|v_{i}\left(p_{v_{i}}\right) ; i\right\rangle$ is the initial product state of the incoming neutrino $v_{i}$ with momentum $p_{v_{i}}$ and the ground state of the target nucleus, and the ket $\left|v_{f}\left(p_{v_{f}}\right) ; f\right\rangle$ is the product state of the outgoing neutrino $v_{f}$ with momentum $p_{v_{f}}$ and one particular excited state of ${ }^{208} \mathrm{~Pb}$ with angular momentum $J_{f}$ and parity $\pi_{f} . H_{\text {eff }}$ is the electro-weak interaction, in the neutral-current sector. After performing all the necessary transformations the cross section is written

$$
\sigma=\frac{G^{2}}{\pi} \sum_{f} E_{v_{f}}^{2} \frac{1}{2} \int_{-1}^{1} d(\cos \theta) M_{\mathrm{Nuc}}
$$

where $G$ is the electro-weak coupling constant, and $M_{\text {Nuc }}$ is the nuclear matrix element. The summation is performed over the complete set of nuclear states of ${ }^{208} \mathrm{~Pb}$, and the integration is done over the angle, $\theta$, between the incoming and outgoing neutrino. To obtain the expression for $M_{\text {Nuc }}$ the interaction $H_{\text {eff }}$ is expanded in powers of the inverse nucleon mass $M_{N}^{-1}$ and in the limit of small momenta, $p_{N} / M_{N} \ll 1$, where $p_{N}$ is the momentum of the nucleon, one obtains:

$$
M_{\mathrm{Nuc}}=\lambda_{\tau} M(\tau)+\lambda_{\sigma \tau} M(\sigma \tau)+\lambda_{\Lambda} M(\Lambda)
$$

The quantities $\lambda_{\tau}, \lambda_{\sigma \tau}$ and $\lambda_{\Lambda}$ depend on the momentum of the outgoing neutrino and contain the nucleon form factors [12-14]. For the isospin-dependent operators the contribution to the nuclear transition probability is given by [15]

$$
\begin{aligned}
M(\tau) & =\left|\left\langle f\left|\tau_{0} e^{i q r}\right| i\right\rangle\right|^{2} \\
& =\frac{4 \pi}{2 J_{i}+1} \sum_{l}\left|\left\langle J_{f}^{\pi}\left\|\sum_{k} \tau_{0}(k) i^{l} j_{l}\left(q r_{k}\right) Y_{l}\left(\hat{\mathbf{r}}_{\mathbf{k}}\right)\right\| J_{i}^{\pi}\right\rangle\right|^{2},
\end{aligned}
$$

and the spin-isospin-dependent part they are given by the matrix element

$$
\begin{aligned}
M(\sigma \tau)= & \left|\left\langle f\left|\sigma \tau_{0} e^{i q r}\right| i\right\rangle\right|^{2} \\
= & \frac{4 \pi}{2 J_{i}+1} \sum_{l, K} \mid\left\langle J_{f}^{\pi} \| \sum_{k} \tau_{0}(k) i^{l} j_{l}\left(q r_{k}\right)\right. \\
& \left.\times\left[Y_{l}\left(\hat{\mathbf{r}}_{\mathbf{k}}\right) \times \sigma(k)\right]^{(K)} \| J_{i}^{\pi}\right\rangle\left.\right|^{2} .
\end{aligned}
$$

The tensor part is written

$$
\begin{aligned}
M(\Lambda)= & \left(\frac{5}{6}\right)^{2} \frac{4 \pi}{2 J_{i}+1} \sum_{l, l^{\prime}, K}(-1)^{l / 2-l^{\prime} / 2+K} \\
& \times \sqrt{(2 l+1)\left(2 l^{\prime}+1\right)}\left(\begin{array}{ccc}
l & l^{\prime} & 2 \\
0 & 0 & 0
\end{array}\right)\left\{\begin{array}{ccc}
1 & 1 & 2 \\
l^{\prime} & l & K
\end{array}\right\} \\
& \times\left\langle J_{f}^{\pi} \| \sum_{k} \tau_{0}(k) i^{l} j_{l}\left(q r_{k}\right)\left[Y_{l}\left(\hat{\mathbf{r}}_{\mathbf{k}}\right) \times \sigma(k)\right]^{(K)}|| J_{i}^{\pi}\right\rangle \\
& \times\left\langle J_{f}^{\pi}\left\|\sum_{k^{\prime}} \tau_{0}\left(k^{\prime}\right) i^{l^{\prime}} j_{l}\left(q r_{k^{\prime}}\right)\left[Y_{l^{\prime}}\left(\hat{\mathbf{r}}_{\mathbf{k}^{\prime}}\right) \times \sigma\left(k^{\prime}\right)\right]^{(K)}\right\| J_{i}^{\pi}\right\rangle^{*} .
\end{aligned}
$$

In the above equations, we have adopted the notation and the phase convention given in [15], concerning the matrix elements of tensor operators written in spherical coordinates and for spherical single-particle states.

\section{B. Nuclear structure of ${ }^{208} \mathrm{~Pb}$}

The wave function, $|J M, k\rangle$ of the excited $k$ th state in ${ }^{208} \mathrm{~Pb}$ is represented as a superposition of neutron particle-hole states $\left|n n^{-1} ; J M\right\rangle$ and proton particle states $\left|p p^{-1} ; J M\right\rangle$

$$
\begin{aligned}
|J M, k\rangle= & \sum_{n n^{-1}} A^{(k)}\left(n, J^{\pi}\right)\left|n n^{-1} ; J M\right\rangle \\
& +\sum_{p p^{-1}} B^{(k)}\left(p, J^{\pi}\right)\left|p p^{-1} ; J M\right\rangle .
\end{aligned}
$$

The quantities $A^{(k)}\left(n, J^{\pi}\right)$ and $B^{(k)}\left(p, J^{\pi}\right)$ are the amplitudes to be obtained by a direct diagonalization of the residual twobody interaction, which in this work is the $\delta$-force interaction of Ref. [16].

\section{RESULTS AND DISCUSSION}

\section{A. Nuclear structure: Single-particle basis and nuclear excitations}

The single-particle neutron and proton states, which are included in the single-particle basis used in the calculations, are the harmonic oscillator states listed in Table I. They are 29 proton states and 22 neutron states. The values of the singleparticle energies were taken from Refs. $[10,17]$. The energies of the states $\left(1 h_{9 / 2}\right)_{n},\left(1 h_{9 / 2}\right)_{p}$, and $\left(3 s_{1 / 2}\right)_{p}$ were adjusted so that the lowest part of the ${ }^{208} \mathrm{~Pb}$ spectrum is reproduced. The parameters $u_{0}$ and $u_{1}$ of the $\delta$-force interaction [16] used in our calculations, have been fixed at the values $u_{0}=-23.66 \mathrm{MeV}$ and $u_{1}=23.66 \mathrm{MeV}$, respectively, to reproduce correctly the energy of the first excited $J^{\pi}=3^{-}$state in ${ }^{208} \mathrm{~Pb}$. 
TABLE I. Energy of the single-particle states for neutrons and protons. The values are given in units of $\mathrm{MeV}$.

\begin{tabular}{|c|c|c|c|}
\hline \multicolumn{2}{|c|}{ Neutrons } & \multicolumn{2}{|c|}{ Protons } \\
\hline State (nlj) & Energy & State (nlj) & Energy \\
\hline $3 s_{1 / 2}$ & -11.10 & $1 f_{7 / 2}$ & -11.90 \\
\hline $1 h_{11 / 2}$ & -10.90 & $1 f_{5 / 2}$ & -11.80 \\
\hline $1 h_{9 / 2}$ & -13.80 & $2 p_{3 / 2}$ & -11.70 \\
\hline $2 f_{7 / 2}$ & -11.80 & $2 p_{1 / 2}$ & -11.60 \\
\hline $1 i_{13 / 2}$ & -9.00 & $1 g_{9 / 2}$ & -11.55 \\
\hline $2 f_{5 / 2}$ & -8.07 & $1 g_{7 / 2}$ & -11.50 \\
\hline $3 p_{3 / 2}$ & -8.00 & $2 d_{5 / 2}$ & -12.70 \\
\hline $3 p_{1 / 2}$ & -7.40 & $1 h_{11 / 2}$ & -9.40 \\
\hline $2 g_{9 / 2}$ & -4.00 & $2 d_{3 / 2}$ & -8.40 \\
\hline $1 i_{11 / 2}$ & -3.20 & $3 s_{1 / 2}$ & -9.50 \\
\hline $1 j_{15 / 2}$ & -2.60 & $1 h_{9 / 2}$ & 2.00 \\
\hline $3 d_{5 / 2}$ & -2.50 & $2 f_{7 / 2}$ & -2.91 \\
\hline $4 s_{1 / 2}$ & -1.90 & $1 i_{13 / 2}$ & -2.20 \\
\hline $2 g_{7 / 2}$ & -1.40 & $2 f_{5 / 2}$ & -1.00 \\
\hline $3 d_{3 / 2}$ & -1.30 & $3 p_{3 / 2}$ & -0.70 \\
\hline $1 j_{13 / 2}$ & -1.25 & $3 p_{1 / 2}$ & -0.15 \\
\hline $2 h_{11 / 2}$ & -1.20 & $2 g_{9 / 2}$ & 4.028 \\
\hline $2 h_{9 / 2}$ & -1.15 & $1 h_{11 / 2}$ & 5.434 \\
\hline $3 f_{7 / 2}$ & -1.10 & $1 j_{15 / 2}$ & 5.960 \\
\hline $3 f_{5 / 2}$ & -1.0 & $3 d_{5 / 2}$ & 6.748 \\
\hline $4 p_{3 / 2}$ & -0.90 & $4 s_{1 / 2}$ & 7.843 \\
\hline \multirow[t]{8}{*}{$4 p_{1 / 2}$} & -0.80 & $2 g_{7 / 2}$ & 8.087 \\
\hline & & $3 d_{3 / 2}$ & 8.530 \\
\hline & & $2 h_{11 / 2}$ & 11.390 \\
\hline & & $3 f_{7 / 2}$ & 12.748 \\
\hline & & $1 k_{17 / 2}$ & 14.066 \\
\hline & & $2 h_{9 / 2}$ & 15.964 \\
\hline & & $1 j_{13 / 2}$ & 15.086 \\
\hline & & $2 i_{13 / 2}$ & 18.143 \\
\hline
\end{tabular}

The dimension of the particle-hole basis, for each value of the angular momentum and parity $J^{\pi}$ of the calculated spectrum, are shown in Table II. We have tested the quality of the basis by varying the number of single-particle states in the basis and looking at the convergence of the calculated spectrum against the addition of more single-particle states. The values of $N$ given in this table represent the extension of

TABLE II. Dimension $(N)$ of the configuration-space used in the calculation of excited states with angular momentum and parity $J^{\pi}$ of ${ }^{208} \mathrm{~Pb}$.

\begin{tabular}{lrlr}
\hline \hline$J^{\pi}$ & $\mathrm{N}$ & $J^{\pi}$ & $\mathrm{N}$ \\
\hline $0^{+}$ & 18 & $0^{-}$ & 20 \\
$1^{+}$ & 52 & $1^{-}$ & 55 \\
$2^{+}$ & 80 & $2^{-}$ & 78 \\
$3^{+}$ & 98 & $3^{-}$ & 96 \\
$4^{+}$ & 108 & $4^{-}$ & 103 \\
$5^{+}$ & 108 & $5^{-}$ & 101 \\
$6^{+}$ & 99 & $6^{-}$ & 91 \\
$7^{+}$ & 83 & $7^{-}$ & 75 \\
\hline \hline
\end{tabular}

TABLE III. Energy of the lowest excited states in ${ }^{208} \mathrm{~Pb}$. The experimental values are denoted by $E_{\exp }$ and the calculated ones by $E_{\text {exp }}$. All values are given in units of $\mathrm{MeV}$. The experimental values are taken from Ref. [19].

\begin{tabular}{ccccccccc}
\hline \hline$J^{\pi}$ & $E_{\exp }$ & $E_{\text {calc }}$ & $J^{\pi}$ & $E_{\exp }$ & $E_{\text {calc }}$ & $J^{\pi}$ & $E_{\exp }$ & $E_{\text {calc }}$ \\
\hline $3_{1}^{-}$ & 2.615 & 2.617 & $3_{2}^{-}$ & 4.051 & 2.848 & $6_{1}^{+}$ & 4.424 & 3.705 \\
$5_{1}^{-}$ & 3.198 & 2.904 & $2_{1}^{+}$ & 4.086 & 3.259 & $1_{1}^{-}$ & 4.842 & 3.008 \\
$4_{1}^{-}$ & 3.475 & 3.466 & $5_{4}^{-}$ & 4.180 & 4.050 & $0_{1}^{+}$ & 4.868 & 5.470 \\
$5_{2}^{-}$ & 3.708 & 3.282 & $6_{2}^{-}$ & 4.206 & 4.316 & $5_{1}^{+}$ & 5.193 & 4.847 \\
$6_{1}^{-}$ & 3.920 & 3.921 & $2_{1}^{-}$ & 4.230 & 3.587 & $0_{1}^{-}$ & 5.281 & 5.028 \\
$4_{2}^{-}$ & 3.947 & 3.796 & $3_{3}^{-}$ & 4.255 & 3.754 & $3_{1}^{+}$ & 5.383 & 4.628 \\
$5_{3}^{-}$ & 3.961 & 3.720 & $4_{4}^{-}$ & 4.262 & 4.602 & $1_{1}^{+}$ & 5.844 & 5.521 \\
$4_{3}^{-}$ & 3.995 & 4.209 & $5_{5}^{-}$ & 4.296 & 4.235 & & & \\
$7_{1}^{-}$ & 4.037 & 3.682 & $4_{1}^{+}$ & 4.324 & 3.138 & & & \\
\hline \hline
\end{tabular}

the particle-hole basis needed to saturate the energy-weighted strength of the associated multipole operators [18].

In Table III we show the calculated energies of the lowest portion of the spectrum of ${ }^{208} \mathrm{~Pb}$, together with the experimental values [19]. In general, it is seen that the calculated energies agree acceptably well with the experimental ones, except for the second $J^{\pi}=3^{-}$state and the first $J^{\pi}=4^{+}$state, where the difference in energies are larger than for the other cases. We turn now to the discussion of the structure of the lowest excited states in ${ }^{208} \mathrm{~Pb}$, as a test of the calculations. The largest components of the first excited $3^{-}$state in the neutron-sector are $\left(2 g_{9 / 2} 3 p_{3 / 2}^{-1}\right),\left(1 i_{11 / 2} 2 f_{5 / 2}^{-1}\right),\left(2 g_{9 / 2} 2 f_{5 / 2}^{-1}\right),\left(2 g_{7 / 2} 2 f_{5 / 2}^{-1}\right)$, and $\left(1 j_{15 / 2} 1 i_{13 / 2}^{-1}\right)$, while in the proton sector the main components are $\left(1 h_{9 / 2} 2 d_{3 / 2}^{-1}\right),\left(2 f_{7 / 2} 3 s_{1 / 2}^{-1}\right)$, and $\left(1 i_{13 / 2} 1 h_{11 / 2}^{-1}\right)$. The calculated amplitudes, for these configurations, are quite comparable with the results of Ref. [20], where the RPA model was applied to calculate the lowest spectra of ${ }^{208} \mathrm{~Pb}$, and with those of Ref. [21], where the amplitudes have been obtained within the TDA framework, although in these references the amplitude of the $\left(1 h_{9 / 2} 2 d_{3 / 2}^{-1}\right)$-proton configuration is somehow larger than the one that we have obtained. However, the

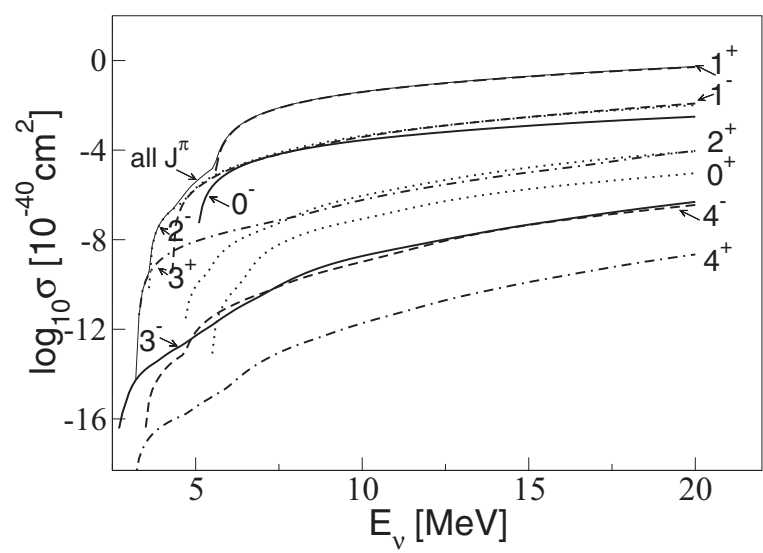

FIG. 1. Contributions to the cross sections of different final states of ${ }^{208} \mathrm{~Pb}$, as a function of the energy of the incoming neutrino $E_{v}<$ $20 \mathrm{MeV}$. The curve labeled "all" is the sum of all partial contributions. 


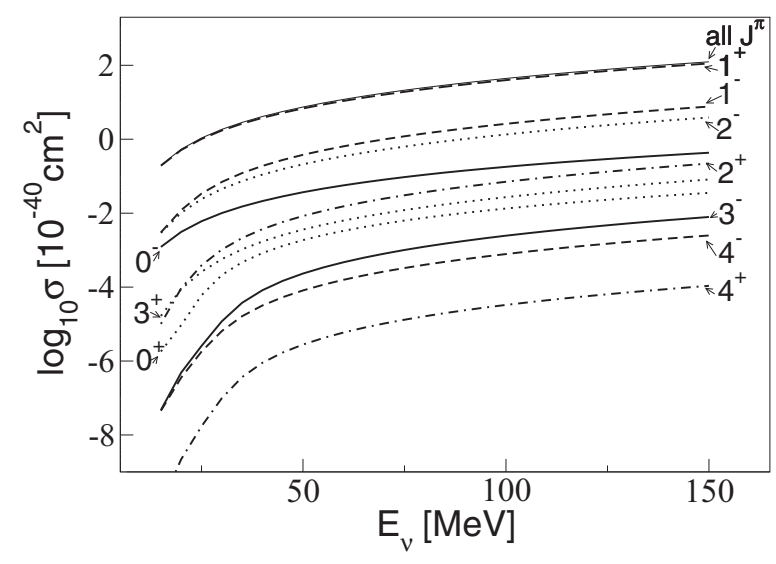

FIG. 2. The same as Fig. 1, for $E_{v}<150 \mathrm{MeV}$.

influence of this difference upon the cross section is minor, as we shall show in Sec. III B.

\section{B. Cross sections}

In Fig. 1 we show the contributions to the cross section, for different multipole states, as a function of the energy of the incoming neutrino, and for $E_{v}<20 \mathrm{MeV}$. Each curve has been obtained by summing on all the eigenstates of a given angular momentum and parity. It is seen from this figure that the set of $J^{\pi}=1^{+}$-states gives the largest contribution to the cross section.

In Fig. 2 we show the components of the cross section, for energies up to $E_{v}=150 \mathrm{MeV}$. Also for this energy range, the dominant contributions are those of the $J^{\pi}=1^{+}$states, although the energy dependence is less pronounced than that for the low-energy domain. In fact, the cross section changes approximately five orders of magnitude, for $5 \mathrm{MeV}<E_{v}<$ $25 \mathrm{MeV}$, and only by one order of magnitude, for $50 \mathrm{MeV}$ $<E_{v}<150 \mathrm{MeV}$.
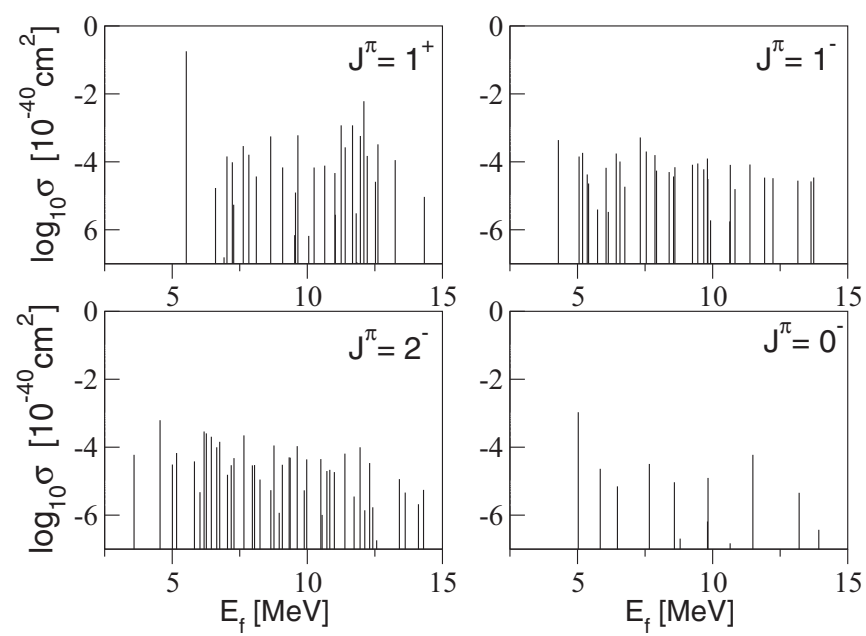

FIG. 3. Contributions to the cross sections, for each eigenstate belonging to the set of states with angular momentum and parity $J^{\pi}=0^{-}, 1^{ \pm}, 2^{-}$, as a function of the energy of the states, and for $E_{v}=15 \mathrm{MeV}$.
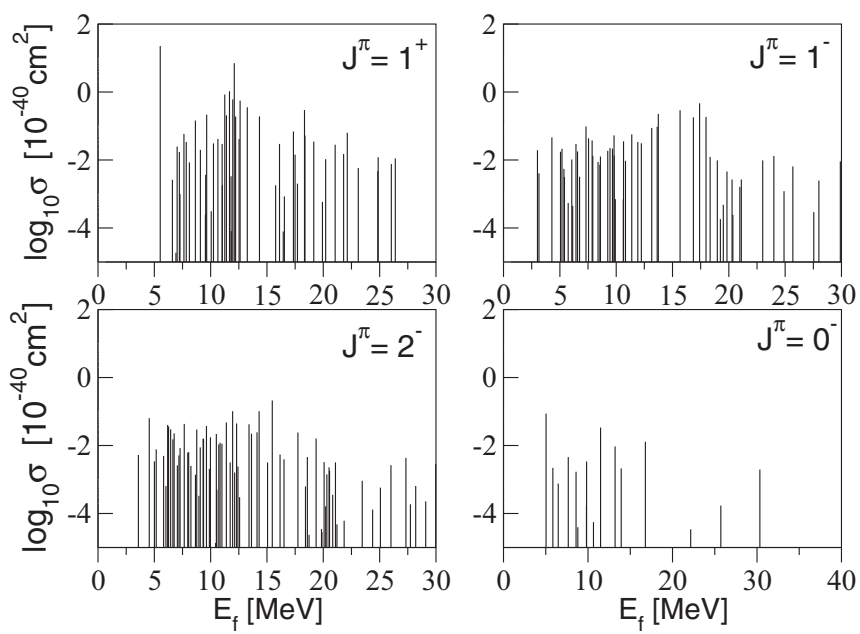

FIG. 4. Contributions to the cross sections, for each eigenstate belonging to the set of states with angular momentum and parity $J^{\pi}=0^{-}, 1^{ \pm}, 2^{-}$, as a function of the energy of the states, and for $E_{v}=95 \mathrm{MeV}$.

This is also seen in Figs. 3 and 4, where the partial contributions to the cross sections from the states of some of the dominant multipolarities are shown as a function of the excitation energy. The results displayed in these figures correspond to the incoming neutrino energies $E_{v}=15 \mathrm{MeV}$ and $E_{v}=95 \mathrm{MeV}$, respectively.

In Table IV we compare the calculated total cross section, for two values of the incident neutrino energy: $E_{v}=15 \mathrm{MeV}$, and $E_{v}=95 \mathrm{MeV}$, with the results available in the literature. The choice of these energies was dictated by the availability of published results. It is seen that the present values are comparable with the values reported earlier, which have been obtained using different approaches to the description of the nuclear structure sector of the calculations. For the case of $E_{v}=15 \mathrm{MeV}$ we have obtained a value, which is approximately twice larger than the other values quoted in the table, while for $E_{v}=95 \mathrm{MeV}$ our value is a bit smaller than the other values, though the overall difference of the results obtained with different interactions is not very significant. This feature is dictated by the double closed-shell structure of the target (and residual) nucleus ${ }^{208} \mathrm{~Pb}$, where the inclusion of dominant particle-hole configurations is more crucial than the particular residual interactions adopted for the calculations. In our case, the results obtained with the $\delta$-force interaction, as it is the case of a separable multipole-multipole interaction [18], reproduced rather satisfactorily the properties of the excited low-lying states of ${ }^{208} \mathrm{~Pb}$.

TABLE IV. Total cross section for two values of incoming neutrino energy $E_{v}$ compared to the result of other works. The values are given in units of $10^{-40} \mathrm{~cm}^{2}$.

\begin{tabular}{lccc}
\hline \hline$E_{v}(\mathrm{MeV})$ & Our calculation & Ref. [7] & Ref. [8] \\
\hline 15 & 0.199 & 0.08 & 0.0798 \\
95 & 38.74 & 47.39 & 41.3 \\
\hline \hline
\end{tabular}


TABLE V. Contributions to the cross sections for different multipolarities, for the incoming neutrino energies $E_{v}=15 \mathrm{MeV}$, second and third columns, and $E_{v}=95 \mathrm{MeV}$, fourth and fifth columns. For each incoming neutrino energy we are listing the energy centroid $\left(E_{\mathrm{c}}\right)$ and the contribution to the cross section. The departure of the values, of the partial cross sections, with respect to the reference power $10^{-40}$ is given in parentheses.

\begin{tabular}{lclrl}
\hline \hline$J^{\pi}$ & $E_{\mathrm{c}}(\mathrm{MeV})$ & $\sigma\left(10^{-40} \mathrm{~cm}^{2}\right)$ & $E_{\mathrm{c}}(\mathrm{MeV})$ & $\sigma\left(10^{-40} \mathrm{~cm}^{2}\right)$ \\
\hline $0^{+}$ & 9.329 & $1.813(-6)$ & 20.084 & $1.175(-2)$ \\
$0^{-}$ & 5.552 & $1.221(-3)$ & 8.511 & $1.606(-1)$ \\
$1^{+}$ & 5.891 & 0.19206 & 7.928 & 35.020 \\
$1^{-}$ & 7.411 & $3.023(-3)$ & 14.042 & 2.282 \\
$2^{+}$ & 9.231 & $1.013(-5)$ & 19.413 & $6.104(-2)$ \\
$2^{-}$ & 7.049 & $2.945(-3)$ & 11.625 & 1.179 \\
$3^{+}$ & 9.353 & $1.664(-5)$ & 16.695 & $2.357(-2)$ \\
$3^{-}$ & 10.083 & $4.719(-8)$ & 24.894 & $2.110(-3)$ \\
$4^{+}$ & 10.978 & $1.278(-10)$ & 26.680 & $2.812(-5)$ \\
$4^{-}$ & 10.367 & $4.531(-8)$ & 22.655 & $6.748(-4)$ \\
\hline \hline
\end{tabular}

In Table $\mathrm{V}$ we show the multipole decomposition of the cross sections, together with the centroids corresponding to each multipolarity, for two values of the incoming neutrino energy $E_{v}$. As said before, the largest contribution is given by the set of $1^{+}$, and it is followed in importance by the contribution from the $1^{-}$and $2^{-}$states. ${ }^{1}$ It is seen that the contribution to the cross section of the set of $3^{-}$states is very small, as compared to the dominant contribution from $1^{+}$ states, and the same applies to the other multipolarities. Next, we have calculated particle-hole excitations in the continuum for each multipolarity. The results are given in Table VI. To perform the calculations we have used Breit-Wigner

\footnotetext{
${ }^{1}$ In the case of the set of $J^{\pi}=1^{-}$states, the contribution coming from the spurious state was partially removed by renormalizing the two-body interaction for this channel. Although the removal of the spurious center of mass motion is not complete, the contribution of this multipolarity to the final cross section is small enough to justify the approach.
}

TABLE VI. Contributions due to continuum $\sigma_{\text {cont }}$ for different multipolarities, for the incoming neutrino energies $E_{v}=15 \mathrm{MeV}$, second column, and $E_{v}=95 \mathrm{MeV}$, third column. The notation is the same as in Table V.

\begin{tabular}{lcc}
\hline \hline$J^{\pi}$ & $\sigma_{\text {cont }}\left(10^{-40} \mathrm{~cm}^{2}\right)$ & $\sigma_{\text {cont }}\left(10^{-40} \mathrm{~cm}^{2}\right)$ \\
\hline $0^{+}$ & $4.019(-12)$ & $2.984(-7)$ \\
$0^{-}$ & $6.203(-10)$ & $6.615(-7)$ \\
$1^{+}$ & $9.271(-7)$ & $4.071(-4)$ \\
$1^{-}$ & $2.909(-5)$ & $1.875(-5)$ \\
$2^{+}$ & $5.947(-5)$ & $8.140(-4)$ \\
$2^{-}$ & $2.060(-9)$ & $5.646(-6)$ \\
$3^{+}$ & $1.308(-5)$ & $9.880(-5)$ \\
$3^{-}$ & $1.563(-6)$ & $2.107(-4)$ \\
$4^{+}$ & $9.668(-10)$ & $6.576(-7)$ \\
$4^{-}$ & $7.643(-7)$ & $5.192(-5)$ \\
\hline \hline
\end{tabular}

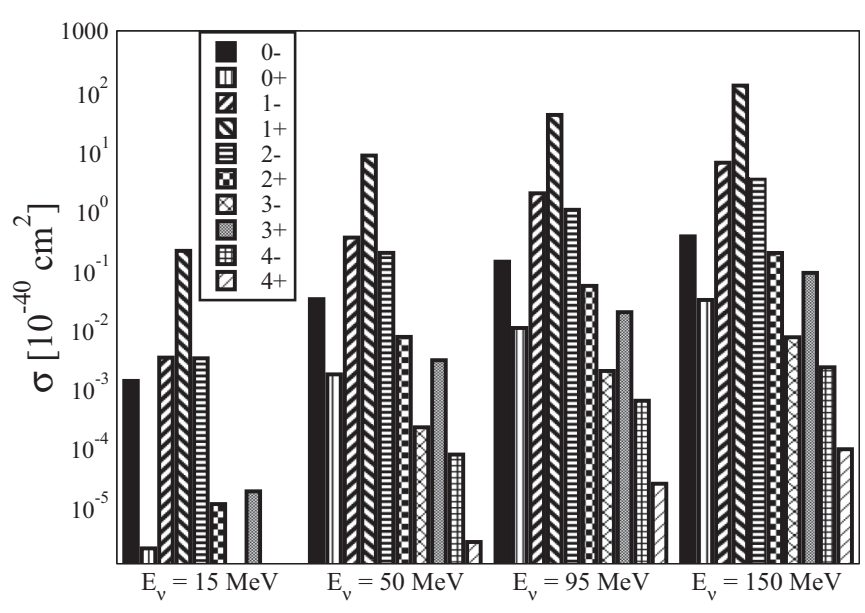

FIG. 5. Multipole decomposition of the contributions to the cross section for different values of incoming neutrino energy.

distribution functions to integrate the partial differential cross section around resonances [22]. As it is seen from these results, the contributions due to these states are much smaller than the ones of the discrete spectrum, a result which resembles that of the charged currents [10]. In order to test the validity of this statement, we have recalculated the cross section for different values of the width $\Gamma$ of the Breit-Wigner functions, for each multipolarity, and found that the results were not affected much by the choice of the width, the values of which were actually fixed at $10 \mathrm{keV}$ and $100 \mathrm{keV}$, respectively.

In Fig. 5 we present the multipole decomposition of the contributions to the cross section for different values of the incoming neutrino energy. From the results shown in this figure, it is observed that the dominance of the contribution of $J^{\pi}=1^{+}$states remains for the considered incoming neutrino energies.

\section{CONCLUSIONS}

In this work we have calculated the contribution of the neutral-current part of the neutrino-lead interaction to the cross section for different values of the energy of the neutrino. We have considered single-particle states with discrete energies, for both neutrons and protons. To obtain the wave functions of the excited states of ${ }^{208} \mathrm{~Pb}$ we have diagonalized a $\delta$-force interaction, with parameters adjusted to reproduce the energy of the first excited state in ${ }^{208} \mathrm{~Pb}$. With these wave functions, we have calculated the cross section for different values of the neutrino energy, and performed a multipole decomposition of it. From the calculated values we conclude that: (i) The value of the cross section for the $\left(v, v^{\prime}\right)$ process is considerably smaller than the value of the cross section of the $\left(v, e^{-}\right)$process. The ratio between these cross sections, at $E_{v}=95 \mathrm{MeV}$, is given by the estimate $\frac{\sigma\left(v, v^{\prime}\right)}{\sigma\left(v, e^{-}\right)} \approx 0.12$. (ii) The dependence of the cross section for neutral current on the incoming neutrino energy is approximately exponential for $E_{v}<40 \mathrm{MeV}$, and it goes almost linearly at higher energies where it saturates at the value $\sigma\left(\nu, v^{\prime}\right) \approx 10^{-38} \mathrm{~cm}^{2}$. (iii) The multipole decomposition of the cross section demonstrates that the dominant contribution 
comes from transition to the states with $J^{\pi}=1^{+}$, with a centroid of the order of $1 \hbar \omega$, a value that is consistent with the activation of the $\tau_{0}$ component of the Gamow-Teller $(\sigma \tau)$ operator. This information may help to understand the behavior of the nuclear response, in this case of ${ }^{208} \mathrm{~Pb}$, to neutrino probes from different sources.

\section{ACKNOWLEDGMENTS}

This work has been supported by the National Research Council (CONICET) of Argentina (Grant PIP 282) and by the ANPCYT of Argentina. The authors are members of the scientific research career of the CONICET.
[1] B. Fields and S. Sarkar in W.-M. Yao et al., J. Phys. G 33, 1 (page 220), (2006) also available at arXiv:astro-ph/0601514 (2006).

[2] W. P. Wright, G. Nagaraj, J. P. Kneller, K. Scholberg, and I. R. Seitenzahl, Phys. Rev. D 94, 025026 (2016).

[3] J. Suhonen and O. Civitarese, Phys. Rep. 300, 123 (1998).

[4] S. Pascoli and S. T. Petcov, Phys. Lett. B 544, 239 (2002).

[5] S. R. Elliott, Phys. Rev. C 62, 065802 (2000).

[6] G. M. Fuller, W. C. Haxton, and G. C. McLaughlin, Phys. Rev. D 59, 085005 (1999).

[7] J. Engel, G. C. McLaughlin, and C. Volpe, Phys. Rev. D 67, 013005 (2003).

[8] E. Kolbe and K. Langanke, Phys. Rev. C 63, 025802 (2001).

[9] T. C. Shantz, M.Sc. Thesis, Laurentian University, School of Graduate Studies, 2010.

[10] O. Civitarese, R. J. Liotta, and M. E. Mosquera, Phys. Rev. C 78, 064308 (2008).

[11] R. J. Blin-Stoyle, Fundamental Interactions and the Nucleus (North-Holland, Amsterdam, 1973).
[12] M. Fukugita, Y. Kohyama, K. Kubodera, and T. Kuramoto, Astrophys. J. 337, L59 (1989).

[13] M. Fukugita, Y. Kohyama, K. Kubodera, and T. Kuramoto, Phys. Rev. C 41, 1359 (1990).

[14] T. Kuramoto, M. Fukugita, Y. Kohyama, and K. Kubodera, Nucl. Phys. A 512, 711 (1990).

[15] A. Bohr and B. Mottelson, Nuclear Structure (Benjamin, Reading, 1969), Vol. 1.

[16] P. Alexa, J. Kvasil, and R. K. Sheline, Phys. Rev. C 55, 3170 (1997).

[17] I. Hamamoto, Phys. Rep. 10, 63 (1974).

[18] A. Bohr and B. Mottelson, Nuclear Structure (Benjamin, Reading, 1975), Vol. 2.

[19] M. J. Martin, Nucl. Data Sheets 108, 1583 (2007).

[20] V. Gillet, A. M. Green, and E. A. Sanderson, Nucl. Phys. A 88, 321 (1966).

[21] W. W. True, C. W. Ma, and W. T. Pinkston, Phys. Rev. C 3, 2421 (1971).

[22] O. Civitarese and M. Gadella, Phys. Rep. 396, 41 (2004). 